Int. J. Contemp. Math. Sciences, Vol. 2, 2007, no. 31, 1549 - 1551

\title{
Splitting Criteria for Vector Bundles on Singular Quadrics
}

\author{
E. Ballico ${ }^{1}$ \\ Dept. of Mathematics \\ University of Trento \\ 38050 Povo (TN), Italy \\ ballico@science.unitn.it
}

\begin{abstract}
Here we give a few splitting criteria for vector bundles on singular quadrics.
\end{abstract}

\section{Mathematics Subject Classification: $14 J 60$}

Keywords: quadric hypersurface; singular quadric; splitting criterion; vector bundles

\section{INTRODUCTION}

For all integers $n, s$ such that $n \geq 2$ and $-1 \leq s \leq n-2$ let $Q_{n, s} \subset$ $\mathbf{P}^{n+1}$ denote an $n$-dimensional quadric hypersurface whose singular locus has dimension $s$. Hence $Q_{n}:=Q_{n,-1}$ is smooth, each $Q_{n, s}$ is integral and for fixed $n, s$ all $Q_{n, s}$ 's are projectively normal. We recall the following facts on the smooth quadric hypersurface $Q_{n}$ ([3], [8], [9], [10], [1]). See $Q_{n-1}$ has a smooth hyperplane section of $Q_{n}$ If $n=2 m$ is even (resp. $n=2 m-1$ is odd), then there are two non-isomorphic (resp. one) vector bundles $S_{n}^{\prime}, S_{n}^{\prime \prime}$ (resp. $\left.S_{n}\right)$ on $Q_{n}$ with rank $2^{m-1}$ such that $S_{2 m}^{\prime}\left|Q_{2 m-1} \cong S_{2 m}^{\prime \prime}\right| Q_{2 m-1} \cong S_{2 m-1}$ and $S_{2 m+1} \mid Q_{2 m} \cong S_{2 m}^{\prime} \oplus S_{2 m}^{\prime \prime}$. These vector bundles $S_{n}, S_{n}^{\prime}$ and $S_{n}$ are called the spinor bundles and we will denote them with $S_{n}$ or just $S$ even if $n$ is not odd. A vector bundle $E$ on $Q_{n}, n \geq 3$, splits (i.e. it is isomorphic to a direct sum of line bundles if and only if $h^{i}\left(Q_{n}, E(t)\right)=0$ for all $t \in \mathbb{Z}$ all $1 \leq i \leq n-1$ and $h^{i}\left(Q_{n}, E(t) \otimes S\right)=0$ and all spinor bundles $S$, all $t \in \mathbb{Z}$ and all $1 \leq i \leq n-2$ ([10], Th. 3.4). When $n=2$ the same vanishings are equivalent to the splitting of $E$ into a direct some of balanced line bundles, i.e. of line bundles $\mathcal{O}_{Q_{2}}(t)$ for some $t \in \mathbb{Z}$. If $\operatorname{rank}(E)$ is low, say $\operatorname{rank}(E)<n$, stronger criteria are true ([6], Th. 2.2 and Th. 3.1). For all integers $n, s, t$ such that $-1 \leq t<s \leq n-2$ see $Q_{n, s}$ has a $(t-s)$-codimensional linear seqction

\footnotetext{
${ }^{1}$ The author was partially supported by MIUR and GNSAGA of INdAM (Italy).
} 
of $Q_{n+t-s, t}$. In particular we may see $Q_{n, s}$ as a linear section of the smooth quadric $Q_{n+s+1,-1}$. set $S_{n, s}:=S_{n+s+1} \mid Q_{n, s}$, where $S_{n+s+1}$ denote any spinor bundle of $Q_{n+s+1}$. Hence $Q_{n, s}$ has one spinor bundle if $n+s+1$ is odd and (a priori) two spinor bundles if $n+s+1$ is even. Here we will prove that the quoted splitting criteria are true also for the singular quadric hypersurfaces $Q_{n, s}$.

Theorem 1. Fix integers $n, s$ such that $0 \leq s \leq n-3$. Let $E$ be a vector bundle on $Q_{n, s}$. E is isomorphic to a direct sum of line bundles if and only if $h^{i}\left(Q_{n, s}, E(t)\right)=0$ for all $t \in \mathbb{Z}$ all $1 \leq i \leq n-1$ and $h^{i}\left(Q_{n, s}, E(t) \otimes S\right)=0$ and all spinor bundles $S$, all $t \in \mathbb{Z}$ and all $1 \leq i \leq n-2$.

Proposition 1. Fix integers $n, s$ such that $0 \leq s \leq n-7$. There is no rank 2 indecomposable vector bundle $E$ on $Q_{n, s}$ such that $h^{i}\left(Q_{n, s}, E(t)\right)=0$ for all $1 \leq i \leq n-2$ and all $t \in \mathbb{Z}$.

Theorem 2. Fix integers $n, s$ such that $0 \leq s \leq n-5$. A vector bundle $E$ on $Q_{n, s}$ with $\operatorname{rank}(E) \leq n-s-3$ splits if and only if $h^{i}\left(Q_{n, s}, E(t)\right)=0$ for all $2 \leq i \leq n-2$ and all $t \in \mathbb{Z}$ and $h^{1}\left(Q_{n, s}, E(t) \otimes S\right)=0$ for all $t \in \mathbb{Z}$ and all spinor bundles $S$ on $Q_{n, s}$.

Remark 1. Use induction on $s$, the corresponding vanishings on $Q_{n+s+1}$ and standard short exact sequences to get $h^{i}\left(Q_{n, s}, \mathcal{O}_{Q_{n, s}}(t)\right)=h^{i}\left(Q_{n, s}, S_{n, s}(t)\right)=0$ for all $1 \leq i \leq n-1$ and $h^{n}\left(Q_{n, s}, \mathcal{O}_{Q_{n, s}}(t)\right)=0$ for $t \geq-n+1$.

Remark 2. Assume $0 \leq s \leq n-2$. Here we will check that $\operatorname{Pic}\left(Q_{n, s}\right) \cong \mathbb{Z}$ and that $\mathcal{O}_{Q_{n, s}}(1)$ is a generator of $\operatorname{Pic}\left(Q_{n, s}\right)$. Indeed, taking linear sections it is easy to reduce to the case $n=2$ and $s=0$ (the quadric cone in $\mathbf{P}^{3}$. In this case that the Hirzebruch surface $F_{2}$ is the blowing-up of the vertex of $Q_{2,0}$ and that a line of $Q_{n, s}$ is a Weil divisor, but not a Cartier divisor (or use [2], Ex. V. Ex. 2.9, for some help).

Proof of Theorem 1. Remarks 1 and 2 gives the "only if" part. Fix $E$ satisfying all vanishings and let $Q_{n-s-1} \subset Q_{n, s}$ a general codimension $s+1$ linear sections. Since $S_{2 m+1} \mid Q_{2 m} \cong S_{2 m}^{\prime} \oplus S_{2 m}^{\prime \prime}$ and $S_{2 m}^{\prime}\left|Q_{2 m-1} \cong\right| S_{2 m-1}$, we get that $S_{n, s} \mid Q_{n-s-1}$ is isomorphic to a direct sum of spinor bundles of $Q_{n-s-1}$. A few standard exact sequences and Remark 2 give $h^{i}\left(Q_{n-s-1},\left(E \mid Q_{n-s-1}\right)(t)\right)=$ 0 for all $t \in \mathbb{Z}$ all $1 \leq i \leq n-s-2$ and $h^{i}\left(Q_{n, s},\left(E \mid Q_{n-s-1}\right)(t) \otimes S\right)=0$ and all spinor bundles $S$, all $t \in \mathbb{Z}$ and all $1 \leq i \leq n-s-3$. Since $n-s-1 \geq 2$, [10], Th. 3.4, implies $E \mid Q_{n-s-1} \cong \oplus_{i=1}^{r} \oplus_{Q_{n-s-1}}\left(a_{i}\right), r:=\operatorname{rank}(E)$, for some $a_{i} \in \mathbb{Z}$. Set $F:=\oplus_{i=1}^{r} \oplus_{Q_{n, s}}\left(a_{i}\right)$. Hence $\operatorname{Hom}(F, E)$ is isomorphic to a direct sum of copies of twists of $E$. Hence $h^{i}\left(Q_{n-t, t}, \operatorname{Hom}(F, E)(t)\right)=0$ for all $t \in \mathbb{Z}$ and all $1 \leq i \leq$ $n-t-1$ for all $0 \leq t \leq s-1$. The vanishing of $h^{1}\left(Q_{n-s, 0}, \operatorname{Hom}(F, E)(-1)\right)$ gives that any isomorphism $\sigma: E \mid Q_{n-s-1} \rightarrow \oplus_{i=1}^{r} \oplus_{Q_{n-s-1}}\left(a_{i}\right)$ lifts to a morphism $\tau: E \mid Q_{n-s, 0} \rightarrow \oplus_{i=1}^{r} \oplus_{Q_{n-s, 0}}\left(a_{i}\right) . \tau$ is a morphism between vector bundles of the same rank whose restriction to each point of an effective ample divisor is an isomorphism. Hence $\tau$ is an isomorphism. If $s=0$, then we get $F \cong E$ and 
hence we are done. If $s>0$ we use $s-1$ further steps, each of them equal to the one just done.

Proof of Proposition 1. Assume the existence of such vector bundle $E$. See $Q_{n-s-1}$ as a linear section of $Q_{n, s}$. The usual standard exact sequences give $h^{i}\left(Q_{n-s-1}, E(t) \mid Q_{n-s-1}\right)=0$ for all $1 \leq i \leq n-s-3$ and all $t \in \mathbb{Z}$. Since $n-s-1 \geq 6$, [6], part 2 of Cor. 3.3, $E \mid Q_{n-s-1}$ is decomposable. As in the proof of Theorem 1 we get that $E$ is decomposable, contradiction.

Proof of Theorem 2. Use the proof of Proposition 1 and [6], Th. 2.2.

It would be nice to extend as much as possible the approach with monads given in [4],[5],[6], [7] and [7] to singular quadrics.

We work over an algebraically closed field $\mathbb{K}$ with $\operatorname{char}(\mathbb{K}) \neq 2$.

\section{REFERENCES}

[1] V. Ancona and G. Ottaviani, Some applications of Beilinson's theorem to projective spaces and quadrics, Forum Math. 3 (1991), 157-176.

[2] R. Hartshorne, Algebraic Geometry, Springer, Berlin, 1977.

[3] H. Knörrer, Cohen-Macaulay modules of hypersurfaces singularities I, Invent. Math. 88 (1987), 153-164.

[4] N. Mohan Kumar, C. Peterson and A. P. Rao, Monads on projective spaces, Manuscripta Math. 112 (2003), no. 2, 183-189.

[5] N. Mohan Kumar, A. P. Rao and G. V. Ravindra, Arithmetically Cohen-Macaulay bundles on hypersurfaces, axXiv:math.AG/0507161.

[6] F. Malaspina, Monads and vector bundles on quadrics, preprint.

[7] F. Malaspina, Monads and rank 3 vector bundles on quadrics, preprint.

[8] G. Ottaviani, Critéres de scindage pour les fibrés vectoriels sur les grassmanniens et les quadriques, C. R. Acad. Sci. 305 (1987), 257-260.

[9] G. Ottaviani, Spinor bundles on quadrics, Trans. Amer. Math. Soc. 307 (1988), no. 1, 301-316.

[10] G. Ottaviani, Some extensions of Horrocks criterion to vector bundles on Grassmannians and quadrics, Annali Mat. Pura Appl. (IV) 155 (1989), 317-341.

\section{Received: June 24, 2007}

